\title{
Modélisation de la gestion du projet de recherche via l'approche processus
}

\author{
Mohamed Amine M'BARKI \\ Professeur d'enseignement supérieur à l'Université Abelmalek Essâdi \\ Responsable de l'UFR Management des organisations \\ Ecole Nationale de Commerce et de Gestion- Tanger - Maroc
}

\section{Hajar CHEKIRI}

Etudiante chercheuse à l'Ecole Nationale de Commerce et de Gestion - Tanger - Maroc

\section{Résumé:}

Le présent article relate une expérience réalisée, dans le cadre de l'UFR[1] Management des organisations, pour la formalisation de la recherche au niveau du DESA[2] Management Industriel et ce en proposant un modèle type piloté via l'approche processus.

Certes, la recherche scientifique ne peut, aujourd'hui, qu'être associée à la qualité. Cette dernière est traduite, dans notre modèle, en une démarche bien décrite et soigneusement évaluée. Le choix de la démarche qualité comme cadre de pilotage de la recherche émane d'un bon esprit d'innovation et de créativité, et notre cartographie en témoigne.

Nous avons essayé d'associer à cette approche processus un système documentaire ayant pour objectif d'assurer la traçabilité et de capitaliser un savoir faire, désormais, intéressant.

La mesure était aussi l'un des éléments sur lesquels nous nous sommes penchés. Convaincus de l'importance de l'amélioration continue, des indicateurs de qualité et de gestion ont servis de base pour notre système de mesure.

Simple dans sa conception et riche dans ses apports, ce modèle vient renforcer ce pont entre les questionnements théoriques et les aspects pratiques de la recherche et de proposer un cadre unifié de travail. 
Mots clés : Projet de recherche, approche processus, cartographie, indicateur, amélioration continue. 


\section{Introduction:}

Les pays en voie de développement s'engagent dans la construction des bases de leurs entrées dans la nouvelle économie du savoir qui aura comme piliers majeurs ; la recherche et l'innovation. Il s'agit donc de relever le défi de la qualité de la recherche scientifique dans ses pays.

Un nouveau moyen pour mesurer la richesse des nations est devenu désormais connu, il s'agit bien évidemment du capital immatériel, ce dernier ne peut être rentable sans une démarche bien ficelée de la recherche scientifique.

L'encouragement de l'évolution scientifique et du développement technologique, passe par l'instauration de nouvelles méthodes de gestion de la recherche scientifique. Ces méthodes facilitent le transfert du savoir de l'université vers l'entreprise et contribuent au développement local, régional, national et/ou international.

En effet, on est appelé à attribuer à la recherche scientifique une valeur qui résulte non seulement de la qualité des méthodes déployées mais aussi de l'engagement des acteurs, tout cela dans le cadre d'une démarche qualité bien décrite mais aussi soigneusement appliquée. Le but sera donc de partir des principes de la recherche en gestion et d'établir un nouveau modèle qui permet de renforcer ce pont entre questionnements théoriques et aspects pratiques. Le choix de la démarche qualité comme cadre de pilotage des projets de recherche ne peut être un simple choix sélectif mais plutôt un choix motivé par un esprit d'innovation et créativité.

Le présent document relate une expérience réalisée dans le même sens. Il s'agit d'un essai de modélisation du projet de recherche via l'approche processus. Ce modèle a été conçu pour faciliter mais aussi pour rendre efficiente la recherche dans le cadre du module «management de la qualité » pour les étudiant inscrits en DESA[3] dans le domaine du management industriel. L'objectif était de définir les activités à mener par ces étudiants chercheurs et la manière dont elles seront pilotées.

\section{Cadre général :}

Cet essai de modélisation est le fruit d'une longue réflexion mais aussi des efforts entretenus pour réussir l'application de l'approche processus dans le cadre de la gestion du projet de recherche. D'un point de vue pédagogique, le projet de modélisation permet de mieux concrétiser les acquis en matière du management de la qualité, puisque le projet est réalisé dans le cadre du module "management de la qualité » enseigné aux étudiants du DESA Management industriel. Ce projet permet donc de bâtir un pont solide et fiable entre les acquis théorique et leur champ d'application. D'un point de vue individuel, le présent modèle vient répondre aux motivations personnelles de chaque étudiant chercheur. En laissant à l'écart les anciennes pratiques de recherche, cet essai de modélisation œuvre pour décrire avec soin les activités à mener pour chaque étape du projet de recherche. Il s'agit donc d'un point de repère (exemple du modèle d'échéancier) offert au chercheur dans une optique de benchmark entre les différents étudiants, chose qui renforce les résultats escomptés. D'un autre point de vue, cette fois-ci tourné vers l'extérieur, ce projet de modélisation se présente ouvert aux attentes des parties prenantes. Le travail scientifique du chercheur doit faire l'objet d'une production scientifique publication, cette dernière n'est permise que si cette publication est conforme à un ensemble de critères, l'écoute de l'extérieur est donc primordiale à ce niveau. A cet effet, le présent modèle considère les besoins des parties prenantes comme point de départ et envisage, par conséquent, une évaluation interne et des actions correctives recommandées lors des séances des états d'avancements des projets de recherche. 


\section{Objectifs :}

La mise en place de ce modèle de gestion du projet de recherche répond à l'obligation à laquelle doit se tenir le chercheur pour accomplir son parcours, elle est aussi vêtue d'une grande volonté de faciliter la tâche au chercheur à fin de savourer le plaisir de la découverte scientifique, elle est enfin encouragée par un engagement sérieux de la part des différents acteurs concernés. A cet égard, les objectifs définis pour cette tentative de modélisation sont les suivants :

- Fournir une plate forme pour l'élaboration d'un projet de recherche (initialisation, planification, réalisation et suivi, évaluation du projet);

- Organiser, coordonner l'activité de recherche entre les différents intervenants dans une unité de recherche et cerner le rôle et la mission de chaque acteur et ce en parcellisant le travail;

- Capitaliser les expériences et les savoirs en matière de recherche en management et faire bénéficier les parties intéressées;

- Viser la continuité en offrant la bonne démarche méthodologique et en instaurant l'esprit scientifique.

- Souligner l'importance de l'organisation du projet de recherche dans une Unité de Formation et de Recherche (UFR) ou dans une Ecole Doctorale;

- Structurer et formaliser la recherche dans le cadre du module Management de la Qualité ;

La finalité primordiale est de réussir la recherche dans un cadre régit par une bonne sélection de l'information, un sens de responsabilité et d'engagement, un esprit scientifique et méthodique et une écoute attentive des attentes des parties intéressées.

\section{Méthodologie:}

Le présent travail ambitionne, en se basant sur à un ensemble des références bibliographiques traitant l'approche processus, de projeter l'application de ladite approche au niveau de la recherche scientifique. Notre valeur ajoutée consiste à adapter ce modèle qui s'applique au niveau des entreprises pour le cas de la gestion du projet de recherche. .

Nous nous sommes intéressés aux choix des outils à intégrer dans notre modèle. Il s'agissait donc d'un choix sélectif, orienté vers une plus grande simplification et une meilleure compréhension (cartographie, logigramme, PDCA...).

L'implication de nos parties intéressées (étudiants chercheurs) dans l'élaboration du modèle était la pièce maîtresse de sa réussite. Un effort de communication considérable a été déployé pour garantir la participation de nos étudiants (feed back, critiques, autoévaluation...). 


\section{Acteurs / Responsabilités :}

Ce modèle de gestion du projet de recherche ne peut s'avérer réussi sans l'engagement parfait des différents acteurs. Une définition de l'acteur et de sa responsabilité est à la base de l'approche processus appliquée pour la conception de ce modèle (voir la fiche de description des processus).

Les acteurs intervenant dans le cadre de ce modèle de gestion du projet de recherche sont les suivants :

- $\quad$ Le porteur de projet : il s'agit de l'étudiant chercheur, des thésards en management, des praticiens et des enseignants chercheurs qui effectuent une recherche.

Le porteur de projet a des responsabilités[4] à assumer dans le cadre de sa relation avec l'encadrant. Il est tenu de maintenir l'encadrant informé de ses activités, il doit planifier ses sessions de travail avec l'encadrant en lui transmettant les documents utiles par mail ou sur support papier. Ainsi, il est tenu de mettre au courant son encadrant des problèmes ou des opportunités qui pourraient causer des modifications au niveau du plan d'activités.

- L'encadrant / le directeur de recherche: il s'agit dans notre cas du responsable du module «Management de la Qualité ». Il offre à l'étudiant chercheur non seulement le soutien mais aussi l'aide pour évaluer les pistes de recherche choisis. L'encadrant conseille et suggère à partir de l'état d'avancement de l'étudiant.

- Le responsable de la coordination: ce dernier assume la responsabilité de réussir l'application du présent modèle de recherche et ce en cherchant des pistes fiables de communication et en veillant sur le respect des instructions du travail et sur une utilisation correcte des formulaires de recherche. Le responsable de coordination informe les étudiants, en coordination avec le responsable du module, du planning des séances d'états d'avancement des projets de recherche et prend note des écarts détectés par rapport au modèle d'échéancier mis à la disposition des étudiants.

\section{Les clés du pilotage du projet de recherche :}

Le pilotage du projet de recherche n'est pas une tâche facile. La bonne gestion du projet de recherche demande de la rigueur mais aussi de la flexibilité. Les éléments clés de cette gestion sont les suivants :

- $\quad$ Le choix du sujet et l'effort d'encadrement ;

- $\quad$ La durée de la recherche ;

- $\quad$ Les sessions de travail (Suivi et améliorations);

- L'organisation du travail (ateliers de recherche);

- Les ressources matérielles et financières ;

- $\quad$ La base documentaire (références bibliographiques et webographiques) ;

- La nature de relation avec le monde professionnel. 


\section{Pourquoi une approche processus [5]?}

Il peut exister un très grand nombre de démarches visant à gérer un projet de recherche. Contrairement aux autres démarches, celle axée sur l'approche processus permet de fournir des résultats immédiats et beaucoup plus mesurables. L'approche processus réside en une démarche d'analyse importante qui présuppose un cadre rigoureusement établi caractérisé par une définition précise des résultats à atteindre.

L'approche processus désigne, dans notre cas, l'application d'un système de processus pour la gestion du projet de recherche, ainsi que l'identification des interactions et le management de ces processus. Cette approche est transversale dans la mesure où elle ne découle pas de la responsabilité d'un seul acteur mais qui traverse différents centres de responsabilités, chose qui permet un effet de synergie et d'apprentissage. L'approche processus permet l'analyse et la description de chaque processus et oblige donc à définir sa finalité et les activités qui le composent. L'approche processus favorise aussi une mesure objective des dysfonctionnements lors des états d'avancement des projets de recherche et permet de proposer des actions correctives et ce dans une optique d'amélioration continue

La conception du modèle de recherche est assise sur une approche de communication entre les différents acteurs. Il en résulte pour chacun, dans le cadre de son activité, une meilleure compréhension des objectifs et des contraintes.

Le schéma suivant présente l'intérêt de l'approche processus appliquée pour la conception du modèle :

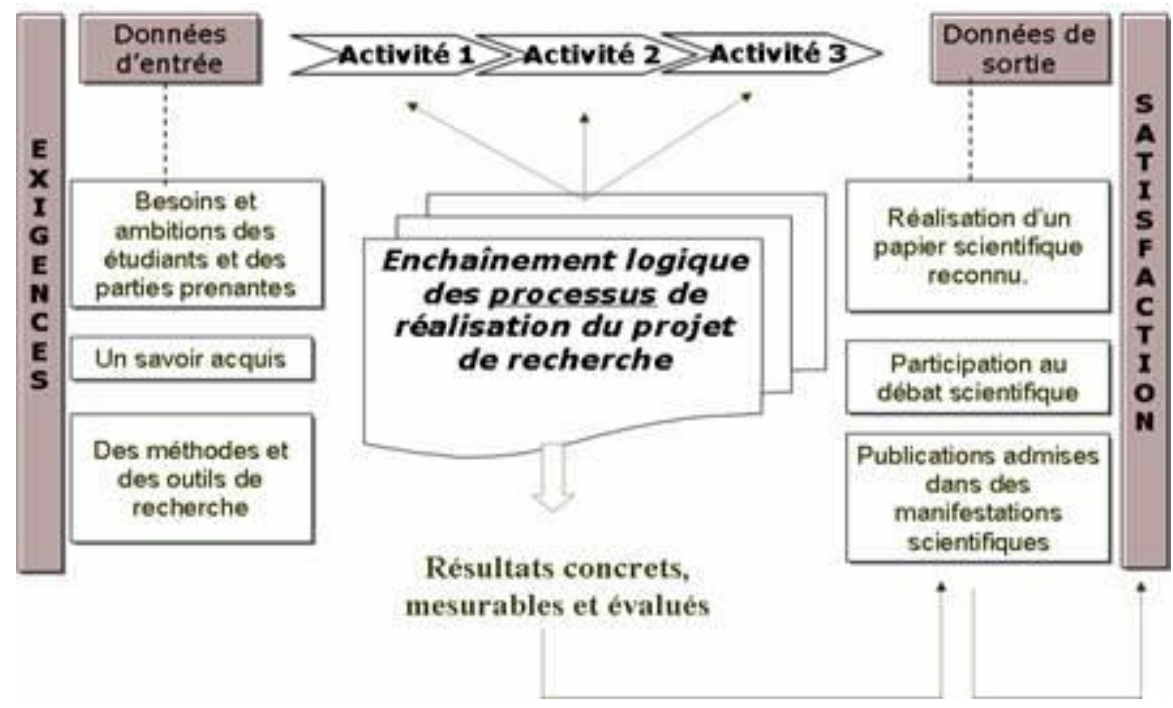

Figure 1 : Intérêt de l'approche processus appliquée pour la conception du modèle.

En partant des exigences (ou besoins) exprimées par les parties prenantes et en passant par un processus de transformation des données d'entrée en données de sorties, l'objectif final sera de satisfaire le client final et/ou les autres parties prenantes.

La réalisation du projet de recherche est considéré comme étant un méga processus qui englobe plusieurs processus, dont chacun est composé d'un ensemble d'activités. La satisfaction du client final sera obtenue via des résultats concrets, mesurables et évalués. Il s'agit donc d'un long enchaînement logique de processus de transformation des données d'entrée (l'idée du projet de recherche, les besoins et ambitions des chercheurs et des parties 
prenantes, le savoir acquis, les méthodes et outils de travails, les motivations et les ambitions personnelles) en des données de sorties (des publications/communication admises dans des manifestations et des revues scientifiques).

\section{La cartographie des processus :}

La cartographie des processus est un outil qui permet de schématiser l'ensemble des processus identifiés et leurs interactions. Elle permet de visualiser une information descriptive et d'attacher des documents de travail tels que procédures, instruction de travail ... Elle permet de représenter les liens entre les processus. La définition des processus de transformation des données d'entrée et données de sorties permet d'élaborer la cartographie de processus suivante (figure 2) :

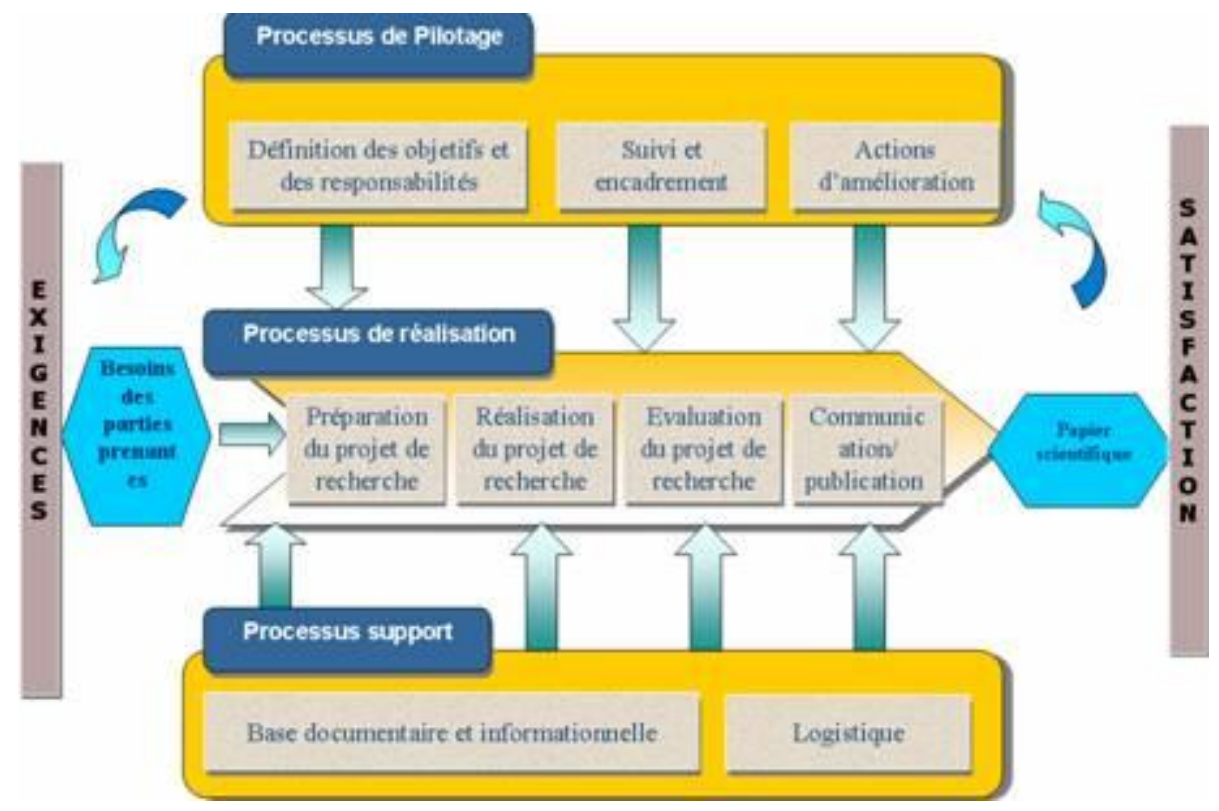

Figure 2 : la cartographie des processus

Notre cartographie de processus conçue dans ce projet est composé de trois catégories de processus : processus de pilotage, processus opérationnel (ou de réalisation) et les processus support. Les différents types de processus définis sont représentés dans le tableau 1:

\begin{tabular}{|c|c|l|}
\hline $\begin{array}{c}\text { Catégorie } \\
\text { du processus }\end{array}$ & $\begin{array}{c}\text { Code du } \\
\text { processus }\end{array}$ & \multicolumn{1}{|c|}{ Intitulé } \\
\hline \multirow{2}{*}{ Pilotage } & PP1 & Définition des objectifs et des responsabilités \\
\cline { 2 - 3 } & PP2 & Suivi et actions d'amélioration \\
\hline \multirow{3}{*}{ Opérationnel } & PR1 & Préparation du PR \\
\cline { 2 - 3 } (Réalisation) & PR2 & Réalisation du PR \\
\cline { 2 - 3 } & PR3 & Évaluation du PR \\
\cline { 2 - 3 } & PR4 & Communication/ publication du papier de recherche \\
\hline
\end{tabular}




\begin{tabular}{|l|l|l|}
\multirow{3}{*}{ Support } & PS1 & Base documentaire et informationnelle \\
\cline { 2 - 3 } & PS2 & Logistique \\
\hline
\end{tabular}

Tableau 1 : les différents types de processus élaborés

La codification des processus revêt un caractère essentiel, dans la mesure où elle facilite leur gestion et permet une meilleure visibilité en ce qui concerne les documents rattachés à chaque processus.

La première tâche qui s'impose au niveau de l'identification des processus, c'est leur identification. Il s'agit à ce niveau de [6] :

- Borner le processus : c'est à dire préciser clairement où il commence et où il finit ;

- $\quad$ Nommer le processus : Cette dénomination doit être suffisamment claire pour qu'il n'y ait aucune ambiguité quant au sujet et au domaine traité. Il est indispensable donc que la dénomination soit courte et si possible qu'elle induise les limites du processus ;

- $\quad$ Ecrire précisément la ou les finalité (s) du processus (produits, service...);

- Situer le processus dans son environnement interne et externe (processus amont, processus aval, liens entre processus adjacents);

- Décrire les différentes activités au sein du processus et les relier entre elles

- $\quad$ Fixer le calendrier (préciser les dates clés, les points d'avancements, etc...)

- Déterminer les indicateurs de mesure pertinents de la performance de chaque activité.

\begin{tabular}{|c|c|c|c|c|c|}
\hline $\begin{array}{l}\text { Intitulé du } \\
\text { processus }\end{array}$ & $\begin{array}{l}\text { Processus } \\
\text { fournisseur }\end{array}$ & Entrées & Clients & Sorties & $\begin{array}{l}\text { Indicateur } \\
\text { (Durée/calendrier) }\end{array}$ \\
\hline $\begin{array}{c}\text { Préparation du } \\
\text { projet }\end{array}$ & $\begin{array}{l}\text { Définition des } \\
\text { objectifs et des } \\
\text { responsabilités }\end{array}$ & $\begin{array}{l}\text { Idée du } \\
\text { thème, grille }\end{array}$ & $\begin{array}{l}\text { Directeur de } \\
\text { recherche }\end{array}$ & Fiche de projet & $\begin{array}{l}\text { - Nombre de références } \\
\text { bibliographiques définies } \\
\text { - La pertinence / } \\
\text { universalité / originalité de la } \\
\text { problématique } \\
\text { - } 50 \text { jours }\end{array}$ \\
\hline $\begin{array}{c}\text { Réalisation du } \\
\text { projet }\end{array}$ & $\begin{array}{l}\text { Préparation du } \\
\text { projet de } \\
\text { recherche }\end{array}$ & $\begin{array}{l}\text { Fiche de } \\
\text { projet }\end{array}$ & $\begin{array}{l}\text { Directeur de } \\
\text { recherche }\end{array}$ & $\begin{array}{l}\text { Résultats } \\
\text { concrets } \\
\text { Rapport }\end{array}$ & $\begin{array}{l}\text { - Coût total pour la } \\
\text { réalisation du projet de } \\
\text { recherche; } \\
\text { - Durée globale de la } \\
\text { réalisation du projet } \\
\text { recherche; } \\
-\quad 110 \text { jours }\end{array}$ \\
\hline $\begin{array}{c}\text { Évaluation } d u \\
\text { projet }\end{array}$ & $\begin{array}{l}\text { Réalisation du } \\
\text { projet de } \\
\text { recherche }\end{array}$ & $\begin{array}{l}\text { Résultats } \\
\text { concrets } \\
\text { Rapport }\end{array}$ & $\begin{array}{l}\text { Directeur de } \\
\text { recherche }\end{array}$ & $\begin{array}{l}\text { Projet validé à } \\
\text { communiquer }\end{array}$ & $\begin{array}{l}\text { - La qualité de l'échantillon } \\
\text { choisi et sa représentativité } \\
\text { - Qualité de rédaction du } \\
\text { rapport; } \\
\text { - Objectifs atteints par } \\
\text { rapport aux objectifs fixés; } \\
\text { - } 20 \text { jours }\end{array}$ \\
\hline
\end{tabular}




\begin{tabular}{|c|l|l|l|l|l|}
$\begin{array}{c}\text { Communication } \\
\text { et publication du } \\
\text { papier de } \\
\text { recherche }\end{array}$ & $\begin{array}{l}\text { Evaluation du } \\
\text { projet de } \\
\text { recherche. }\end{array}$ & $\begin{array}{l}\text { Projet validé } \\
\text { à }\end{array}$ & $\begin{array}{l}\text { - Comité } \\
\text { communiquer } \\
\text { d'un } \\
\text { colloque }\end{array}$ & $\begin{array}{l}\text { - Papier de } \\
\text { recherche }\end{array}$ & $\begin{array}{l}\text { - Nombre de } \\
\text { communications }\end{array}$ \\
& & $\begin{array}{l}\text { - Presse } \\
\text { spécialisée }\end{array}$ & $\begin{array}{l}\text { Communication } \\
\text { dans un } \\
\text { colloque }\end{array}$ & - Nombre de publications. \\
& & $\begin{array}{l}\text { - Publication } \\
\text { dans une revue }\end{array}$ & \\
\hline
\end{tabular}

Tableau 2 : Tableau résumant les caractéristiques des processus opérationnels

La description détaillée des processus a pour élément essentiel la définition du client de chaque processus, ainsi que les entrées et les sorties y rattachés. Le tableau qui suit résume pour chaque processus opérationnel, ses clients, ses données d'entrée ainsi que ses éléments de sortie.

Pour chaque processus une «fiche de processus[7]» est établie pour permettre une meilleure description. Cette fiche contient les éléments suivants : l'intitulé du processus, le responsable du processus, l'objet du processus, le client du processus, les éléments d'entrée, les éléments de sortie, les indicateurs de performance, les moyens et ressources, les étapes du processus et les documents associés.

A titre d'illustration, la fiche de description du processus de préparation du projet de recherche est la suivante :

\begin{tabular}{|c|c|}
\hline $\begin{array}{l}\text { 1. Intitulé du processus : préparation du } \\
\text { projet de recherche }\end{array}$ & $\begin{array}{l}\text { 2. Responsable du processus: L'étudiant } \\
\text { chercheur }\end{array}$ \\
\hline $\begin{array}{l}\text { 3. Objet du processus: définir les étapes } \\
\text { à suivre pour préparer le projet de recherche }\end{array}$ & $\begin{array}{l}\text { 4. Client du processus: Le directeur de } \\
\text { recherche }\end{array}$ \\
\hline $\begin{array}{l}\text { 5. Éléments d'entrée: idée, thème, } \\
\text { objectifs perso et pédagogiques }\end{array}$ & $\begin{array}{l}\text { 6. Éléments de sortie: Formulaire de projet de } \\
\text { recherche }\end{array}$ \\
\hline $\begin{array}{l}\text { 7. Indicateur de performance: } \\
\text { - } \quad \text { Nombre de références bibliographiques } \\
\text { définies } \\
\text { - La pertinence / universalité / originalité } \\
\text { de la problématique }\end{array}$ & $\begin{array}{l}\text { 8. Moyens, Ressources: } \\
\text { - } \quad \text { Internet } \\
\text { - } \quad \text { livres disponibles à la bibliothèque } \\
\text { - } \quad \text { Projets déjà réalisés } \\
\text { _- } \quad \text { Guide de projet de recherche }\end{array}$ \\
\hline
\end{tabular}


9. Étapes du processus: Choix du sujet, formulation du titre, lecture documentaire, préliminaire, élaboration du champ problématique et des hypothèse, rédaction d'une proposition de projet de recherche (formulaire), validation du projet

10. Documents associés: Formulaire de projet de recherche

11. Fournisseur /processus amont : Définition des objectifs et des responsabilités

Tableau 3 : la fiche de description du processus

Pour déterminer les indicateurs de mesure et d'évaluation des projets de recherche, il faut porter un regard global sur l'ensemble des processus (présentés sur la cartographie) et décliner ensuite chaque processus en activités. Pour chaque activité il sera indispensable de souligner les critères d'appréciation de sa performance pour sortir enfin de compte avec des indicateurs pertinents.

Les critères d'appréciation induits des différentes activités sont les suivants :

- $\quad$ les objectifs atteints par rapport aux objectifs fixés;

- La pertinence / universalité / originalité de la problématique;

- $\quad$ recherche, documents colloques..);

- la qualité des références bibliographiques de base du lancement du projet de recherche;

- $\quad$ Le bien fondé des hypothèses;

- le nombre des références bibliographiques (ouvrages, thèses, mémoires, articles, papiers de La durée globale de la réalisation du projet de recherche;

- $\quad$ La qualité de rédaction du papier/rapport de recherche;

- $\quad$ Le coût total pour la réalisation du projet de recherche;

- $\quad$ La qualité du questionnaire conçu;

- La qualité de l'échantillon choisi et sa représentativité

Le tableau 4 ci-après présente les indicateurs d'évaluation du projet de recherche[8] classés en trois catégories : la gestions du temps, la qualité/efficacité et la bibliographie. Dans le cadre 
des projets de recherche en Management de la qualité dans le DESA MI, l'objectif associé à chaque indicateur indique la moyenne à atteindre.

\begin{tabular}{|c|c|c|c|c|}
\hline Indicateur & $\begin{array}{l}\text { Appliqué } \\
\text { Oui/ Non }\end{array}$ & Planification (1) & $\begin{array}{l}\text { Réalisation } \\
\text { (2) }\end{array}$ & $\begin{array}{c}(2) /(1) \\
\%\end{array}$ \\
\hline \multicolumn{5}{|c|}{ Gestion du temps } \\
\hline I- Préparation de la fiche de projet & & 50 jours & & \\
\hline I-1 Recherche et investigation théorique & & 30 jours & & \\
\hline I-2 Elaboration de l'espace problématique & & 15 jours & & \\
\hline I-3 Elaboration du champs des pré réponses & & 7 jours & & \\
\hline II- Elaboration du modèle de recherche & & 7 jours & & \\
\hline III- Elaboration de l'espace empirique & & 102 jours & & \\
\hline III- 1 Conception du questionnaire & & 15 jours & & \\
\hline III-2 Test du questionnaire & & 15 jours & & \\
\hline III-3 Administration du questionnaire & & 50 jours & & \\
\hline III-4 Dépouillement et filtrage des réponses & & 15 jours & & \\
\hline III-5 Analyse des résultats & & 7 jours & & \\
\hline IV- Validation des hypothèses & & 7 jours & & \\
\hline V- Rédaction du rapport final & & 15 jours & & \\
\hline VII- Synthèse du travail & & 7 jours & & \\
\hline $\begin{array}{l}\text { VII- Recherche et définition des espaces de } \\
\text { publication }\end{array}$ & & 20 jours & & \\
\hline VII- Communication et pubication & & 15 jours & & \\
\hline \multicolumn{5}{|c|}{ Qualité/ efficacité } \\
\hline Par rapport aux objectifs fixés & & $\begin{array}{l}\text { Objectifs atteints par rapport } \\
\text { aux objectifs fixés }\end{array}$ & & \\
\hline Par rapport à l'éléments d'entrée & & $\begin{array}{l}\text { Pertinence / universalité / } \\
\text { originalité du sujet }\end{array}$ & & \\
\hline Par rapport au coût & & $\begin{array}{l}\text { Coût total pour la réalisation } \\
\text { du projet de recherche }\end{array}$ & & \\
\hline Par rapport au résultat & & $\begin{array}{l}\text { Qualité de rédaction } \mathrm{du} \\
\text { rapport }\end{array}$ & & \\
\hline Par rapport au technique du travail & & $\begin{array}{l}\text { Qualité de l'échantillon choisi } \\
\text { et sa représentativité }\end{array}$ & & \\
\hline par rapport à la méthodologie adoptée & & $\begin{array}{l}\text { Qualité du questionnaire } \\
\text { conçu }\end{array}$ & & \\
\hline \multicolumn{5}{|c|}{ Bibliographie } \\
\hline Références & & 10 à 20 & & \\
\hline Ouvrages & & 3 à 4 & & \\
\hline Articles & & 3 à 5 & & \\
\hline Thèses & & 1 à 2 & & \\
\hline Colloques/ Séminaires & & 2 à 3 & & \\
\hline Webographie & & 3 à 5 & & \\
\hline Fiches de lecture et synthèses & & 5 à 12 & & \\
\hline
\end{tabular}




\section{Tableau 4 : Les indicateurs d'évaluation}

\section{Étapes du processus global du projet de recherche :}

L'élaboration de la cartographie des processus a été précédée par une étape d'identification des étapes du principal processus relatif à la réalisation du projet de recherche. Cette description du processus est faite dans une vision «d'amélioration continue », elle se compose donc des étapes suivantes :

- Le choix du sujet et de l'encadrant: L'étudiant chercheur peut être très influencé en discutant avec d'autres chercheurs ou en lisant des rapports de travaux déjà réalisés, chose qui donne souvent naissance à une nouvelle problématique chez l'étudiant chercheur. Cette nouvelle problématique l'engage sur une nouvelle thématique de recherche. En effet, le choix du sujet émane non seulement de la volonté du chercheur mais aussi d'une bonne connaissance du cadre référentiel relatif au domaine de recherche. Par conséquent, le chercheur doit choisir soigneusement son sujet de recherche tout en veillant sur le respect de certaines règles, à savoir ; l'originalité du sujet, sa pertinence, son universalité et sa faisabilité. Lorsqu'il s'agit d'un sujet déjà existant, l'étudiant chercheur doit entretenir un grand potentiel d'innovation pour approfondir le sujet. Le choix du sujet est accompagné par la fixation des objectifs qui enchaîne sur la définition de la problématique centrale.

Le chercheur est appelé à choisir son directeur de recherche (encadrant) avant de se lancer dans la réalisation proprement dite. L'étudiant chercheur est confronté parfois à des choix difficiles, le directeur de recherche peut être dans ce cas d'une très grande utilité et ce en le conseillant, l'orientant et parfois en lui présentant sa propre expertise. La relation entre l'étudiant chercheur et son encadrant doit être régie par un grand sens de responsabilité et un engagement à fond. L'étudiant chercheur doit connaître les responsabilités à honorer auprès de son directeur de recherche, et ce dernier ne doit économiser aucun effort de soutien et d'encadrement.

- L'élaboration du formulaire du projet de recherche : L'étudiant chercheur ne s'arrête pas au niveau de la définition de son thème de recherche. Il est tenu de détailler les points à traiter et d'expliciter la méthodologie de travail. A ce niveau, une première version doit être élaborée comme étant une proposition du projet de recherche à remettre au directeur de la recherche. Cette première proposition, faite sous forme d'un formulaire doit présenter avec précision la problématisation. A cet égard, L'étudiant chercheur doit formuler avec soin sa problématique centrale, ses questions dérivées, son hypothèse principale et ses hypothèses sous-jacentes. Il est également tenu d'expliquer son instrumentation méthodologique en lançant un «prés-plan de travail » sur lequel il précise la durée de chaque étape de son travail.

- La validation de la proposition du projet de recherche : le formulaire du projet de recherche demeure une proposition tant que le directeur de recherche ne donne pas son accord et son approbation. Lorsque le projet n'est pas validé, le directeur de recherche peut prévoir des actions d'amélioration tout en encourageant le porteur de projet à refaire sa proposition de projet de recherche.

- Le déroulement des sessions du travail : après validation de sa proposition de projet de recherche, l'étudiant chercheur est tenu de préparer un planning de travail en tenant compte des engagements de son directeur de recherche. Il s'agit donc de prévoir des sessions périodiques de travail, dans lesquelles il présente son état d'avancement dans le projet et sollicite les remarques de son encadrant. L'étudiant chercheur doit être ouvert aux critiques de son encadrant et doit être prêt à assurer continuellement des actions correctives. Les sessions de travail peuvent prendre la forme d' «entretiens de recherche »[9] qui doivent être gérés.

- La validation du projet de recherche et sa communication: La réalisation du projet de recherche doit aboutir à la validation des résultats et à la rédaction d'une ébauche de papier 
assez détaillée qui met en relief ces résultats. Le directeur de recherche doit procéder à une première évaluation du projet de recherche. Il peut se baser, dans le cadre de son évaluation, sur plusieurs critères[10], par exemple :

1. La mise à jour des théories ;

2. L'intérêt du sujet, son importance ;

3. L'approche inédite ou l'approfondissement majeur d'un sujet existant ;

4. La pertinence de la démarche en égard à son objet ;

5. L'articulation des idées ;

6. La progression de la pensée ;

7. La mise en perspective ou en contexte du sujet ;

8. L'usage et le respect des sources citées et consultées ;

9. La qualité de la langue (grammaire, syntaxe, vocabulaire) ;

10. La structure du texte : introduction, développement et conclusion ;

11. La présentation claire et complète des tableaux.

Pour chaque critère, des sous-critères peuvent être envisagés, ce qui permet au directeur de recherche de donner son appréciation générale sur l'article (fruit du projet de recherche). A ce niveau, l'étudiant chercheur doit passer à l'étape de la publication. De ce fait, il doit rester attentif au différents opportunités de communication (congrès, colloques, revues scientifiques...). Le logigramme suivant décrit sommairement les étapes de réalisation du projet de recherche : 


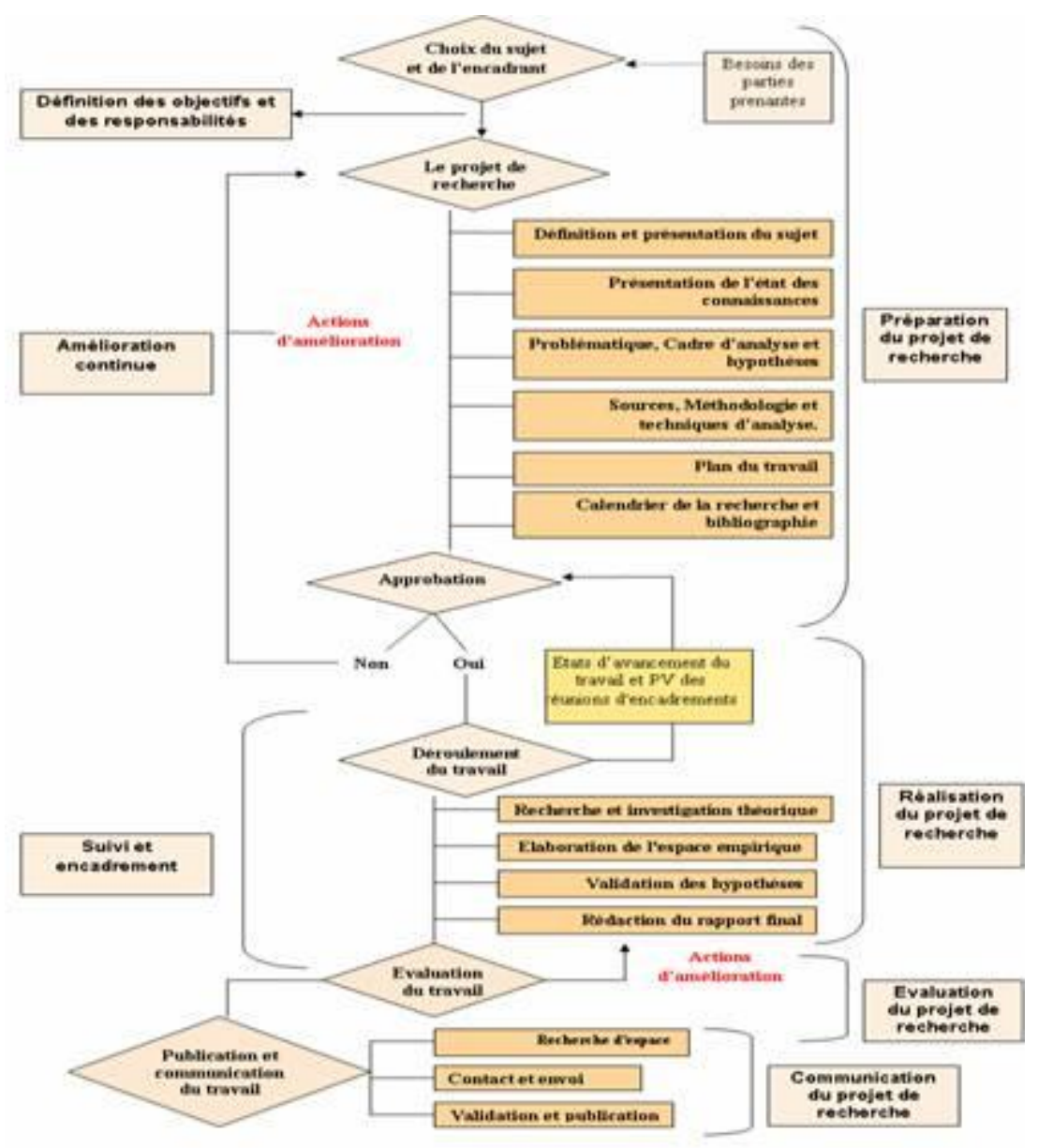

Figure 3 : logigramme du macro processus de réalisation

Toujours dans une optique d'amélioration continue, les étapes du processus « Suivi et actions d'amélioration » ont été schématisés en faisant appel à un des outils qualité, les plus répandus, à savoir de PDCA[11]. Notre roue de Deming sera donc baptisée «PDCA du projet de recherche). Les shèmas suivant présentent les quatre axes du PDCA (planification réalisation - évaluation - amélioration) relatifs au processus du projet de recherche.

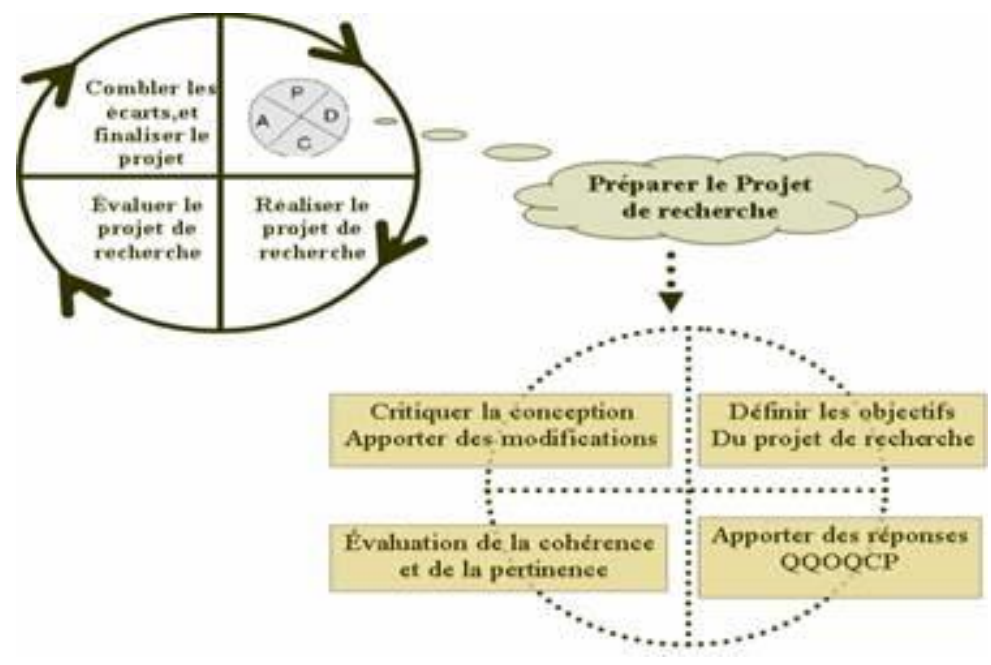

Figure 4 : PDCA de la préparation du projet de recherche 
D'après le schéma, la préparation du projet de recherche passe par la définitions des objectifs du projet de recherche (comme étape de planification), l'opérationnalisation de ces objectifs sera assurée en répondant aux questions (Quoi, Qui, Où, Quand, Comment, Pourquoi) relatives au projet de recherche. L'évaluation de la préparation du projet de recherche passera par l 'évaluation de la cohérence et de la pertinence du sujet de recherche. Lorsque cette étape d'évaluation donne lieu à des critiques, l'étape d'amélioration interviendra pour mettre en place des actions correctives sous forme d'éventuelles modifications.

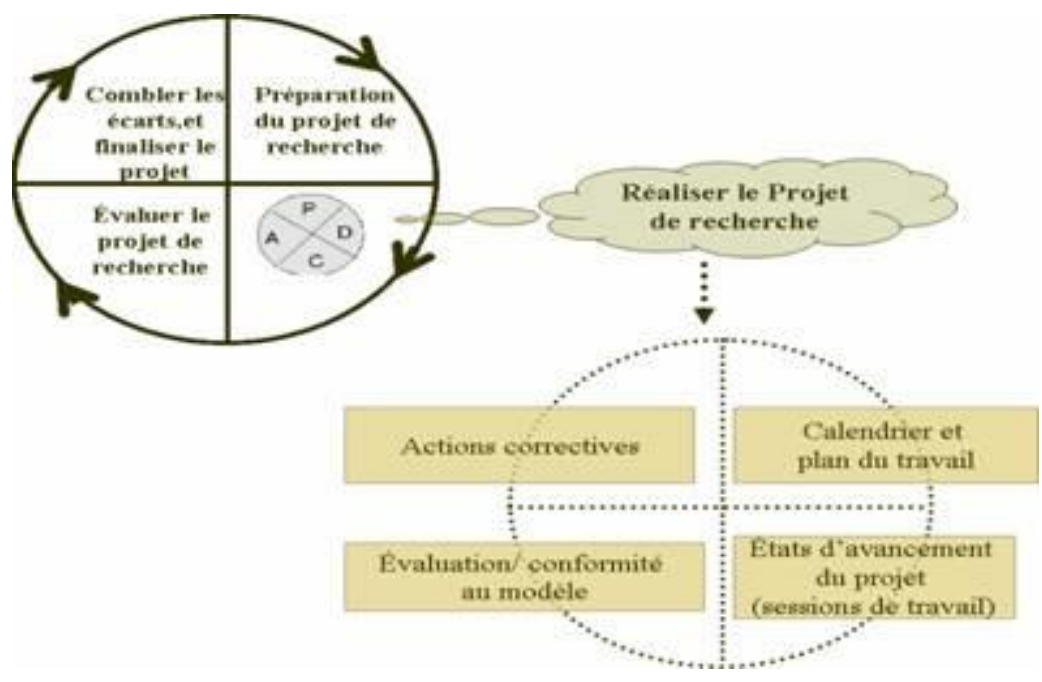

Figure 5 : PDCA de la réalisation du projet de recherche

La planification de la réalisation du projet de recherche est concrétisée par un calendrier de travail qui spécifie les activités à réaliser et le temps accordé à chacune des activités. La réalisation de ce plan de marche passe par la préparation des sessions de travail (états d'avancement). L'étape d'évaluation de la réalisation se base sur la conformité au présent modèle et précisément au modèle d'échéancier standard.



Figure 6 : PDCA de l'évaluation du projet de recherche 
La planification de l'évaluation présuppose la définition des critères d'évaluation, sa réalisation est assurée via la quantification de ces critères. Le contrôle du mode d'évaluation est une étape très importante dans la mesure où elle engage des actions d'améliorations vis à vis du modèle établi.

Pour évaluer le projet de recherche, plusieurs éléments d'évaluation peuvent être retenus. Nous citons :

$\mathrm{Au}$ niveau du fond: Originalité du sujet, cohérence générale, connaissance de la documentation pertinente, maîtrise du sujet, cohérence et rigueur dans l'argumentation, le traitement des sources et données, l'analyse et l'interprétation, dimension personnelle et critique.

Au niveau de la forme : structure formelle (division en chapitres, etc.), style, phraséologie, orthographe et présentation matérielle (régles, normes, etc.).

Dans notre cas, l'évaluation doit prendre en considération la conformité du projet de recherche réalisé par l'étudiant au présent modèle, définit comme standard.

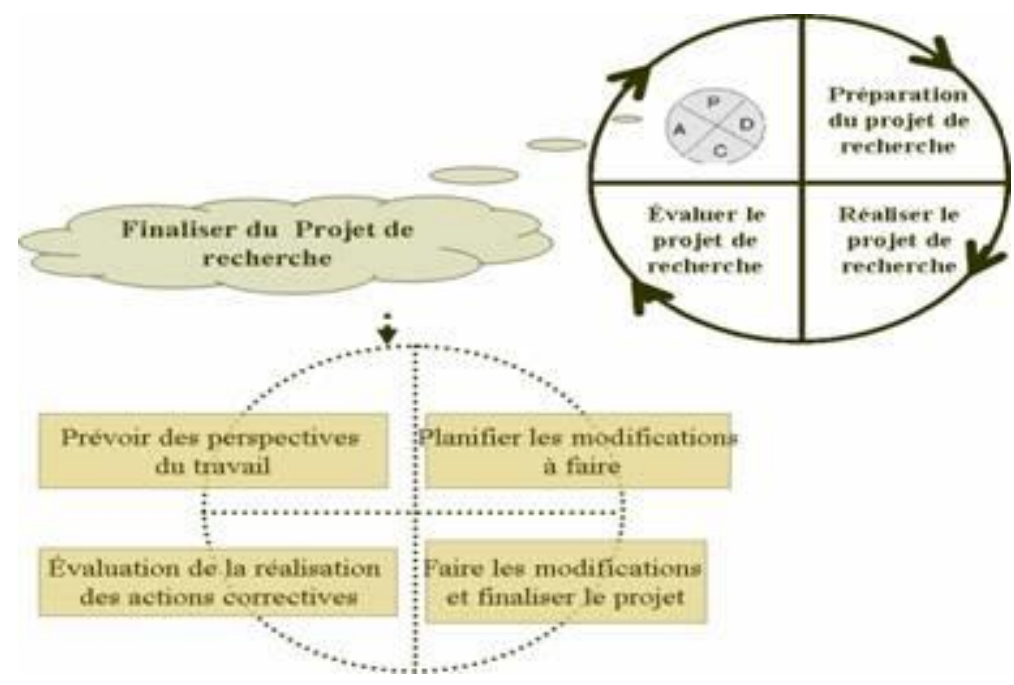

Figure 7 : PDCA de l'amélioration et la finalisation du projet de recherche

L'étape de l'amélioration doit être réalisée avec beaucoup de soin. La planification des modifications à apporter au modèle doit faciliter leur mise en œuvre. Le modèle ne doit pas être figé, au contraire il doit favoriser d'autres pistes d'amélioration et des perspectives futures. 


\section{Les outils de communications :}

La communication est un élément central dans toute organisation composée de plusieurs groupes ou individus. Le circuit d'information doit être fiable, simple et sécurisé. Le choix des outils de communication incombe ici au coordinateur, chargé de transmettre l'information au bon endroit et au bon moment. De ce fait, les outils de communication engagés dans le présent projet sont les suivants :

- $\quad$ Des instructions de travail : il s'agit d'un certain nombre de procédure facilitant la réalisation de certaines tâches ou activités. Ces procédures facilitent non seulement la réalisation mais aussi le contrôle et le suivi par la suite.

- $\quad$ Des modèles - types : il s'agit de quelques formulaires standardisés.

- Des indicateurs de performance : ces indicateurs permettent de communiquer les objectifs de l'évaluation.

- Des règles formelles : ces règles sont le plus souvent prescrites par l'encadrant de la recherche.

- Des e-mails d'information : une boite électronique est crée à cet effet.

\section{Les procédures / instructions de travail[12]:}

Plusieurs procédures sont rédigées afin de formaliser la réalisation de certaines tâches et activités.

\begin{tabular}{|c|l|}
\hline Code & \multicolumn{1}{|c|}{ Intitulé } \\
\hline PRd 01 & Procédure du choix du sujet \\
\hline PRd 02 & Procédure de préparation du projet de recherche \\
\hline PRd 03 & Procédure d'élaboration du calendrier du travail \\
\hline PRd 04 & Procédure des règles des références bibliographiques \\
\hline PRd 05 & Procédure de maîtrise documentaire \\
\hline PRd 06 & Procédure de Préparation d'une fiche de lecture \\
\hline PRd 07 & Procédure du projet de questionnaire/ guide d'entretien \\
\hline PRd 08 & Procédure d'évaluation du projet de recherche \\
\hline PRd 09 & Procédure de communication et de publication \\
\hline
\end{tabular}

\section{Tableau 5 : Les procédures / instructions de travail}

Chaque procédure contient les éléments suivants : Le libellé , le type, les acteurs concernés, les risques encourus, les étapes, les données de sorties ainsi que des observations.

\section{Les modèles - types :}


Plusieurs formulaires de travail sont utilisés afin de formaliser la réalisation de certaines tâches et activités.

\begin{tabular}{|l|l|l|}
\hline \multicolumn{1}{|c|}{ Codes } & \multicolumn{1}{c|}{ Désignation } & \multicolumn{1}{c|}{ Remarques } \\
\hline FO 01 & Formulaire du projet de recherche & Modèle type à respecter \\
\hline FO 02 & Modèle de fiche de lecture & Modèle type à respecter \\
\hline FO 03 & Planning d'encadrement & Modèle type à respecter \\
\hline FO 04 & Modèle d'échéancier & Modèle type à respecter \\
\hline FO 05 & Grille d'évaluation & Grille à remplir \\
\hline
\end{tabular}

Tableau 6 : tableau des Formulaires de travail.

\section{a- Formulaire du projet de recherche}

Le formulaire est composé des élément suivants: le titre complet du projet de recherche, l'objet de la recherche, les mots/ concepts clés, les objectifs et intérêt, l'arborescence problématique, l'arborescence d'hypothèses, la méthodologie, les étapes et la durée du travail, le plan prévisionnel de recherche, la bibliographie provisoire, le coût estimé relatif au projet de recherche et enfin une grille d'évaluation du projet de recherche.

Un Entête standard a été élaboré pour une meilleure gestion de ce formulaire. Cet entête contient le logo de l'établissement, l'intitulé du projet de recherche, la version, le référence et le nombre de page. Sur le pied du document, la succession des révision et précisée avec soin. Le porteur du projet ainsi que le directeur de recherche sont tenus de marquer leur visa sur le document ainsi que leurs éventuelles remarques.

\section{b- Le modèle de la fiche de lecture}

La fiche de lecture rassemble les éléments suivants: une fiche signalétique du document, son contexte, les mots Clés, les objectifs de la recherche, la problématique soulevée, les hypothèses sous-jacentes, un résumé du document, une appréciation du document et une analyse critique du document.

L'entête du document prévoit des information relatives à la nature du document (article, ouvrage, thèse, rapport de colloque...), le titre du document, l'auteur et la source.

\section{c- Le planning d'encadrement}

\begin{tabular}{|c|c|c|c|c|c|c|c|c|c|c|c|c|}
\hline & \multicolumn{2}{|c|}{ L Janvier } & \multicolumn{3}{|c|}{ Féwrier } & \multicolumn{3}{|c|}{ Mars } & \multicolumn{4}{|c|}{ Avril } \\
\hline & 1525 & 3545 & 5565 & 7585 & & $510 \mathrm{~s}$ & 115 & 128 & & & 15155 & 165 \\
\hline Projet 1/ & & & & & & & & & 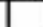 & & . & \\
\hline Projet 2 & & & & & & & & & & & & \\
\hline Projet $Y$ & & & & & . & & & & . & & & \\
\hline Projet 4 I & & & & & & & & & & & & \\
\hline Projet 5/ & & & & & & & & & . & & & \\
\hline Projet 6/ & & & & & & & & & & & & \\
\hline Projet 7 & & & & & & & & & & & & \\
\hline Projet \& & & & & & & & & & 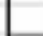 & & & \\
\hline Projet 9 & & & & & & & & & 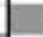 & & & \\
\hline Projet 10l & & & & & & & & & & & & \\
\hline Projet 11: & & & & & & & & & & & & \\
\hline Projet 12 & & & & & & & & & & & & \\
\hline Projet 13. & & & & & & & & & & & & \\
\hline Projet 14: & & & & & & & & & & & & \\
\hline
\end{tabular}




\section{d- Le modèle d'échéancier}

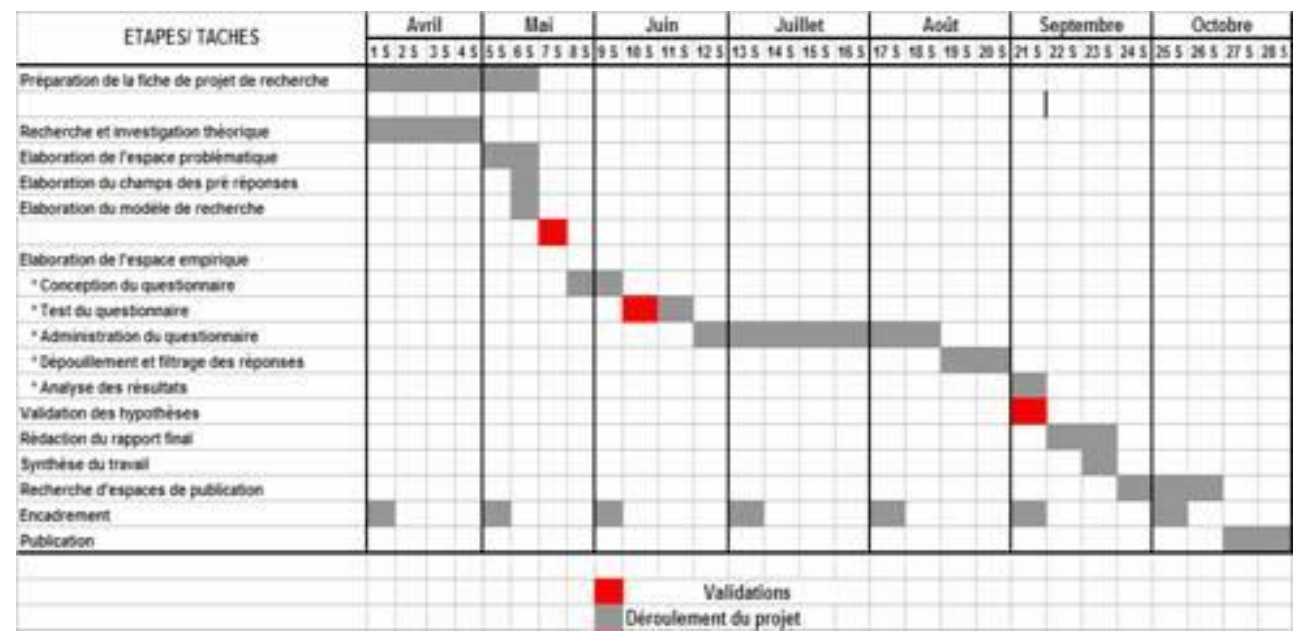

\section{e- La grille d'évaluation}

Il s'agit d'une grille d'évaluation des articles. Cette grille contient les critères d'évaluation suivants: la mise à jour des théories, l'intérêt du sujet, l'importance du sujet, la pertinence de l'approche méthodologique, l'articulation des idées, la progression de la pensée, l'usage et le respect des sources citées et consultées, la qualité de la langue, la structure du texte, la qualité de la présentation. Pour chaque critère d'évaluation, l'étudiant chercheur est tenu d'exprimer une appréciation sous forme d'une auto-évaluation. 


\section{Conclusion:}

L'économie du savoir dans laquelle tous les pays en voie de développement a pour élément catalyseur ; la recherche scientifique. Cette dernière est confrontée, vu le nouveau contexte émergeant, au défi de la qualité.

L'encouragement de la qualité de la recherche ne peut réussir sans une bonne préparation de sa plate-forme. Il s'agira donc non seulement de construire des démarches théoriques mais également leur mise en terrain dans le cadre d'une vision d'amélioration continue.

En se situant par rapport à notre modèle, nos étudiants chercheurs ont formulé plusieurs améliorations afin d'augmenter l'efficacité de nos projets de recherches. Ainsi, la réussite du modèle a été évalué par nos étudiants en s'arrêtant sur le respect des objectifs initiaux, le respect des délais impartis, le respect de l'allocation des ressources et sur la possibilité d'une production scientifique reconnue et sa publication.

En suivant une méthodologie assise sur l'implication et la réactivité de nos étudiants, le présent modèle a été entretenu dans une vision de simplification et de flexibilité, pour tenir compte des propres spécificités de chaque projet de recherche.

Convaincus de l'importance des remarques des parties intéressés et de la nécessité d'une gestion participative de ce modèle de recherche, toutes les remarques et suggestions ont été prises en considération pour l'amélioration du présent travail. La qualité selon notre point de vue ne s'arrête pas au moment de la formalisation, mais elle est plutôt une démarche continue vers la recherche de l'excellence.

[1] Unité de Formation et de Recherche

[2] Diplôme des Etudes Supérieures Approfondies

[3] Diplôme des Etudes Supérieures Approfondies

[4] Pierre Romelaer, Quelques éléments sur les activités du thésard en gestion, papier de recherche $\mathrm{n}^{\circ}$ 79. Centre de recheche pure et appliquée, Université de Paris IX Dauphine, Mai 2002.

[5] Yvon MOUGIN, Processus : les outils d'optimisation de la performance, éditions d'organisation, 2004.

[6] M. CATTAN, N. IDRISSI, P. KNOCKAERT, « maîtriser les processus de l'entreprise, guide opérationnel ». Editions d'organisation. Troisième édition, 2001.

[7] Mohamed Amine M'BARKI, « qu'évalue-t-on quand on met une note à un «projet d'application » dans le module de « gestion de la qualité »CIMQSEF'2005. 
[9] Pierre Romelaer, «Quelques éléments sur les activités du thésard en gestion », Cahier de recherche $n^{\circ} 79$. Mai 2002. CREPA : Centre de recherche pure et appliquée. Université Paris IX Dauphine

[10] Critères d'évaluation tirés de « la grille d'évaluation des articles » élaborée par M. Amine M'BARKI.

[11] André CHARDONNET - Dominique THIBAUDON, «Le guide du PDCA de Deming », Éditions d'Organisation, 2003

[12] Ces procédures font parties du système documentaire. 